\title{
Controlling a chain of integrators with constrained actuation using exponential activation functions
}

\author{
Espen Oland \\ Department of Electrical Engineering \\ UiT - The Arctic University of Norway \\ Narvik, Norway \\ espen.oland@uit.no
}

\author{
Raymond Kristiansen \\ Department of Electrical Engineering \\ UiT - The Arctic University of Norway \\ Narvik, Norway \\ raymond.kristiansen@uit.no
}

\begin{abstract}
This paper shows how to control a chain of integrators with constrained actuation by using exponential activation functions. Specifically, it shows how the control law can be decomposed into several parts that can be activated as they are required, resulting in a simple control law that is able to make the states converge fast to zero.
\end{abstract}

Keywords-Chain of integrators, constrained actuation, exponential activation functions, disturbance rejection.

\section{INTRODUCTION}

$\mathbf{T}$ HE problem of controlling a chain of integrators with limited actuation has received much attention the last decades with many different results, such as nested saturation functions, command-filtered backstepping, modified backstepping, and gain scheduling; c.f. [1]- [7] and references within. With limited actuation, controlling a chain of integrators becomes a challenging problem, as large errors in lower states tend to make the actuators go into saturations, resulting in a loss of control. Solving this problem allows many different systems to be controlled as actuator saturation is inherent in any system, e.g. in [8] the first author shows how to control a quadrotor by controlling a chain of four integrators with constrained actuation by applying the command-filtered backstepping approach.

Schlanbusch and Kristiansen show in [9] how to use exponential gains to apply large gains when errors are large and low gains when the error is small for rotational control of a rigid body. That work has served as an inspiration for this paper, which uses exponential functions to activate parts of the controller as they are required to facilitate control of a chain of integrators with constrained actuation.

The paper is structured as follows: Section II describes the problem statement and how to control a chain of integrators using exponential activation functions. Specifically, it considers standard exponential activation functions, and also an approach where a high-gain feedback is used close to the origin to enable disturbance rejection. Section III shows the performance of the control solution and compares it with nested saturation functions, while Section IV wraps up the paper.

\section{Controlling A Chain of Integrators}

Consider a 4th order chain of integrators as

$$
\begin{aligned}
& \dot{x}_{1}=x_{2} \\
& \dot{x}_{2}=x_{3} \\
& \dot{x}_{3}=x_{4} \\
& \dot{x}_{4}=u,
\end{aligned}
$$

where the objective is to design the control law $u \in[-1,1]$ such that $\mathbf{x}=\left[\begin{array}{llll}x_{1} & x_{2} & x_{3} & x_{4}\end{array}\right]^{\top} \rightarrow \mathbf{0}$. In the unconstrained case, this can easily be achieved using a control law as

$$
u=-k_{1} x_{1}-k_{2} x_{2}-k_{3} x_{3}-k_{4} x_{4}
$$

where $k_{1}, k_{2}, k_{3}, k_{4}$ are four positive gains that must be selected such that the higher level state, $x_{4}$, converges before $x_{3}$ and so on until all states have converged. Figure 1 shows the convergence of the system when $k_{1}=2, k_{2}=20, k_{3}=40$ and $k_{4}=80$. The top subfigure shows the unconstrained case where all errors go to zero. The bottom subfigure shows the case when the actuator signal is bounded between \pm 1 , where it is evident that the control law (5) is unable to make the errors go to zero, such that more advanced control laws are required to solve this problem.

This can be addressed by several different methods. The nested saturation approach by Teel, is perhaps the most wellknown approach for controlling such a chain of integrators, where the control signal can be written as: $u=-\sigma\left(y_{4}+\right.$ $\left.\sigma\left(y_{3}+\sigma\left(y_{2}+\sigma\left(y_{1}\right)\right)\right)\right)$, where $\sigma(\cdot)$ denotes saturation functions bounding the output between two values. The first part stabilizes the $x_{4}$ state through a coordinate transform, while the second part is a nested saturation function allowing the lower level states to converge one at a time. The first observation of this structure is that the available control signal is divided into two objectives, one is to make $x_{4}$ go to zero and one is to make the other states go to zero. In a double integrator case, if the position converges before the velocity error, the position error must diverge to make the velocity error go to zero, such that it is counter-productive to focus on the lower level states before the higher order states have converged. One possible approach 

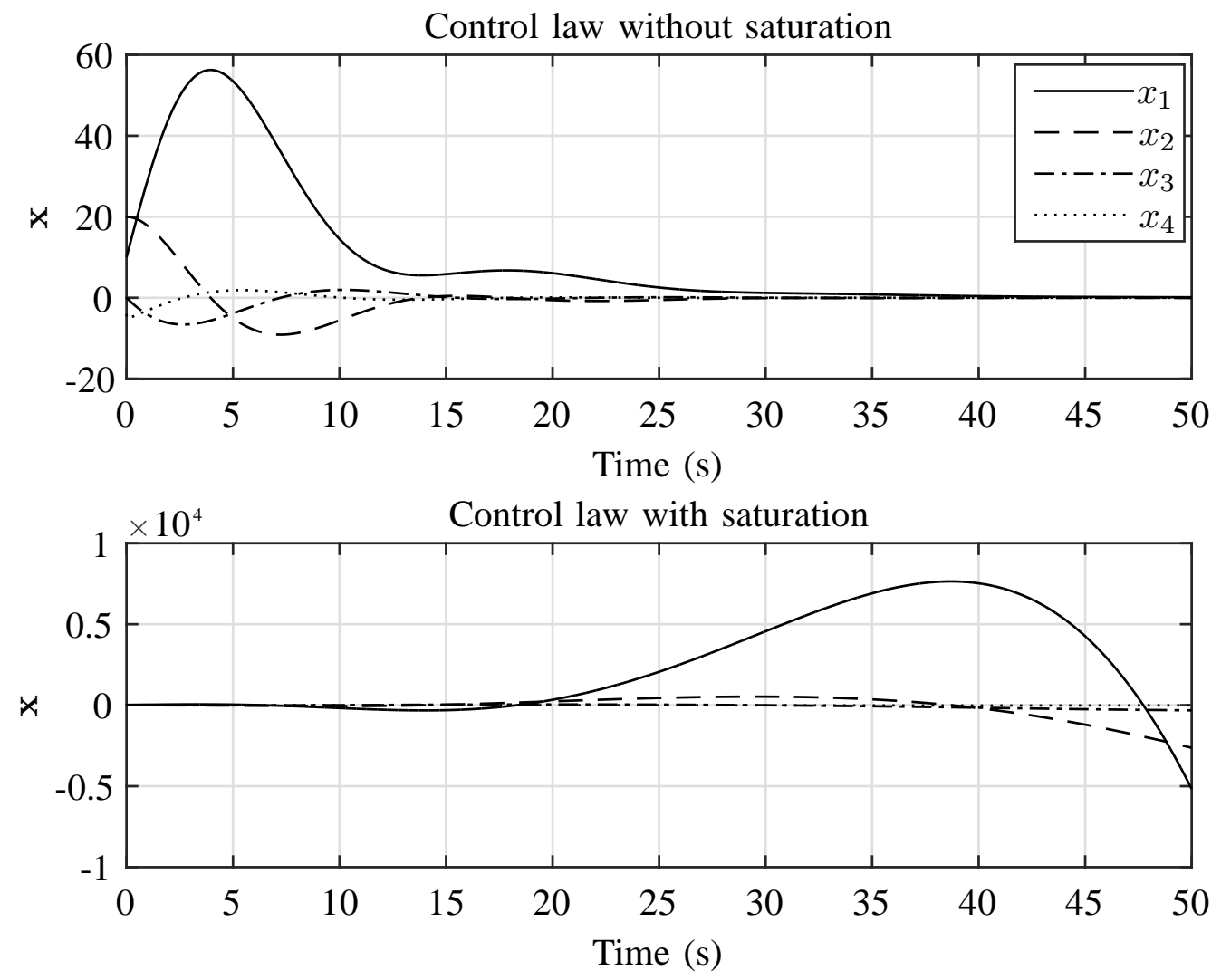

Figure 1. Controlling a chain of integrators with and without saturation. Notice how the states blow up when imposing a constaint on the actuator signal.

to stabilize such a system is through switching, where a control law can be defined as

$$
u= \begin{cases}-k_{4} x_{4}-k_{3} x_{3}-k_{2} x_{2}-k_{1} x_{1} & \text { if } x_{2}, x_{3}, x_{4} \in W \\ -k_{4} x_{4}-k_{3} x_{3}-k_{2} x_{2} & \text { if } x_{3}, x_{4} \in W \\ -k_{4} x_{4}-k_{3} x_{3} & \text { if } x_{4} \in W \\ -k_{4} x_{4} & \text { otherwise }\end{cases}
$$

where $W$ is a bounded set close to the origin. This approach will tackle one problem at a time allowing the states to converge in a successive manner. When $u=$ $-k_{4} x_{4}-k_{3} x_{3}-k_{2} x_{2}-k_{1} x_{1}$, the states $x_{2}, x_{3}$ and $x_{4}$ will be close to zero, such that it is mainly the $x_{1}$ error that governs the control signal. This basic idea can also be made continuous by employing exponential activation functions. Consider a control law as

$$
\begin{aligned}
u= & -k_{1} \exp \left(-c_{1}\left(\left|x_{2}\right|+\left|x_{3}\right|+\left|x_{4}\right|\right)\right) x_{1} \\
& -k_{2} \exp \left(-c_{2}\left(\left|x_{3}\right|+\left|x_{4}\right|\right)\right) x_{2} \\
& -k_{3} \exp \left(-c_{3}\left|x_{4}\right|\right) x_{3} \\
& -k_{4} x_{4}
\end{aligned}
$$

where $c_{1}, c_{2}, c_{3}$ are coefficients that dictate when the lower level stabilizing terms are activated. There are two main advantages with such a control law. Firstly, it is very simple to implement, and secondly, it applies all its control effort to one problem at a time such that it is easy to obtain fast convergence time. The control law can also be extended to facilitate disturbance rejection by adding an additional term to the control law as

$$
\begin{aligned}
u= & -k_{1} \exp \left(-c_{1}\left(\left|x_{2}\right|+\left|x_{3}\right|+\left|x_{4}\right|\right)\right) x_{1} \\
& -k_{2} \exp \left(-c_{2}\left(\left|x_{3}\right|+\left|x_{4}\right|\right)\right) x_{2} \\
& -k_{3} \exp \left(-c_{3}\left|x_{4}\right|\right) x_{3} \\
& -k_{4} x_{4}-k_{5} \exp \left(-c_{4}\left(\left|x_{4}\right|+\left|x_{3}\right|+\left|x_{2}\right|+\left|x_{1}\right|\right) x_{4} .\right.
\end{aligned}
$$

Here, $k_{5}$ can be chosen sufficiently large to reject the disturbances, and $c_{4}$ can be chosen such that it is not activated until the other states have entered the set $W$ to maintain the fast convergence time.

\section{Simulations}

\section{A. 3rd Order Integrator}

To benchmark the approach against the nested saturation function, consider the third order integrator

$$
\begin{aligned}
& \dot{x}_{1}=x_{2} \\
& \dot{x}_{2}=x_{3} \\
& \dot{x}_{3}=u
\end{aligned}
$$



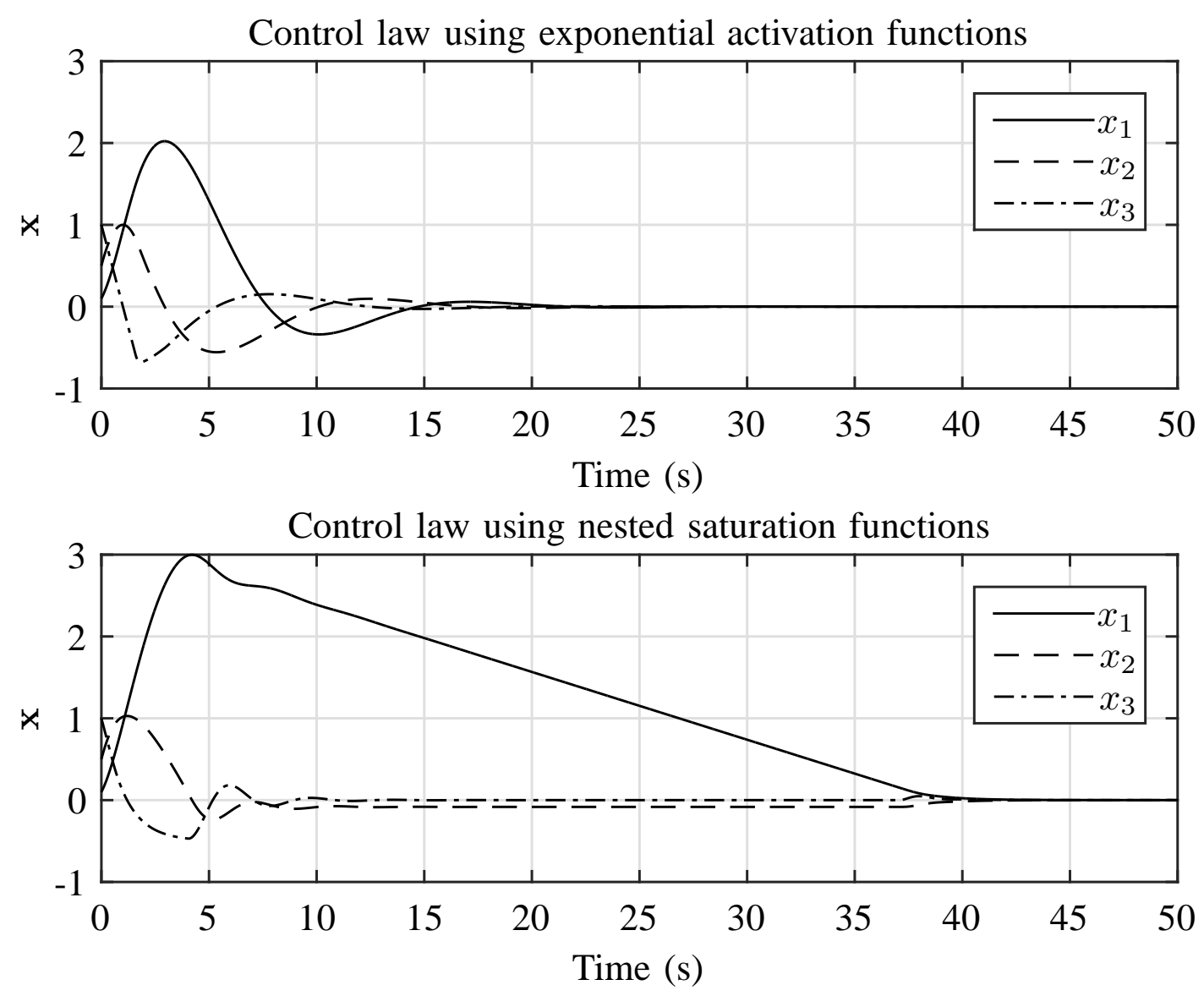

Figure 2. Comparison between the exponential activation controller and the nested saturation controller.

with the initial conditions $x_{1}(0)=0.1, x_{2}(0)=0.5, x_{3}(0)=$ 1 and $|u| \leq 1$. Using nested saturation function, the control law can be designed as

$$
u=-\sigma_{3}\left(y_{3}+\sigma_{2}\left(y_{2}+\sigma_{1}\left(y_{1}\right)\right)\right)
$$

where the transformed coordinates are found as $y_{1}=$ $a_{1} a_{2} a_{3} x_{1}+\left(a_{1}+a_{2}\right) a_{3} x_{2}+a_{3} x_{3}, y_{2}=a_{1} a_{2} x_{2}$ and $y_{3}=a_{1} x_{3}$ with $\left\{a_{1}, a_{2}, a_{3}\right\}=\{1,3,2\}$ [10]. The saturation functions are bounded as $\left|\sigma_{3}(\cdot)\right| \leq 1,\left|\sigma_{2}(\cdot)\right| \leq 0.4990$ and $\left|\sigma_{1}(\cdot)\right| \leq$ 0.2485 , which can be found using the procedure defined in [1] or [10].

Using exponential activation functions, a control law can be designed as

$$
\begin{aligned}
u= & -k_{3} x_{3}-k_{2} \exp \left(-c_{2}\left|x_{3}\right|\right) x_{2} \\
& -k_{1} \exp \left(-c_{1}\left(\left|x_{3}\right|+\left|x_{2}\right|\right) x_{1} .\right.
\end{aligned}
$$

where the gains and coefficients can be chosen as $k_{1}=2, k_{2}=$ $4, k_{3}=8, c_{1}=c_{2}=0.1$. Figure 2 shows the results, where it is evident that with the selected gains and coefficients the new approach is able to obtain faster convergence than the results presented [10]. Note that is possible to improve the performance of both controllers, but the aim of this paper is to highlight the simplicity of this control structure and its applicability to chains of integrators.

\section{B. 4th Order Integrator with Disturbance Rejection}

Now consider the case of a 4th order integrator with a timevarying disturbance similar to (1)-(4), but where $\dot{x}_{4}=u+$ $d(t)$ with $d(t)=0.8 \sin \left(\frac{\pi}{2} t\right)$ and with the initial conditions as $x_{1}(0)=0.8, x_{2}(0)=-0.6, x_{3}(0)=0.5$ and $x_{4}(0)=1$. Consider the controller (6) with the gains $k_{1}=0.1, k_{2}=2$, $k_{3}=20$ and $k_{4}=40$, and $c_{1}=c_{2}=c_{3}=0.1$, and $|u| \leq$ 1. For the disturbance rejection controller (7), the additional parameters are chosen as $k_{5}=2000$ and $c_{4}=2$. Figure 3 shows the convergence of the 4th order integrator with an unknown time-varying disturbance. Both controllers are able to make the errors converge to a bounded set close to the origin, but it is evident that by using a high gain close to the origin reduces the error by about $50 \%$. Note that by selecting $k_{4}=2000$ when not using disturbance rejection results in slower convergence time, such that this represents a method of achieving both fast convergence and disturbance rejection.

\section{CONCLUSION}

This paper has presented a simple control solution for controlling a chain of integrators with constrained actuation. 


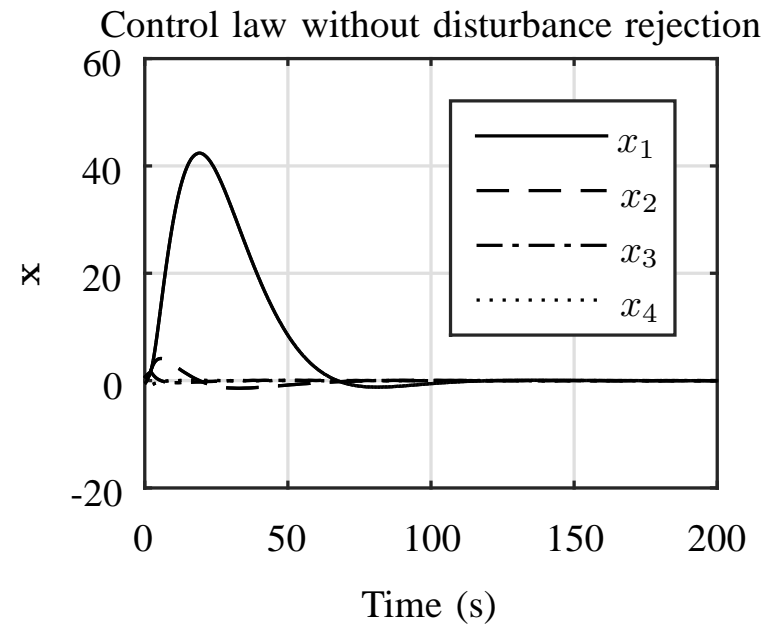

Control law with disturbance rejection

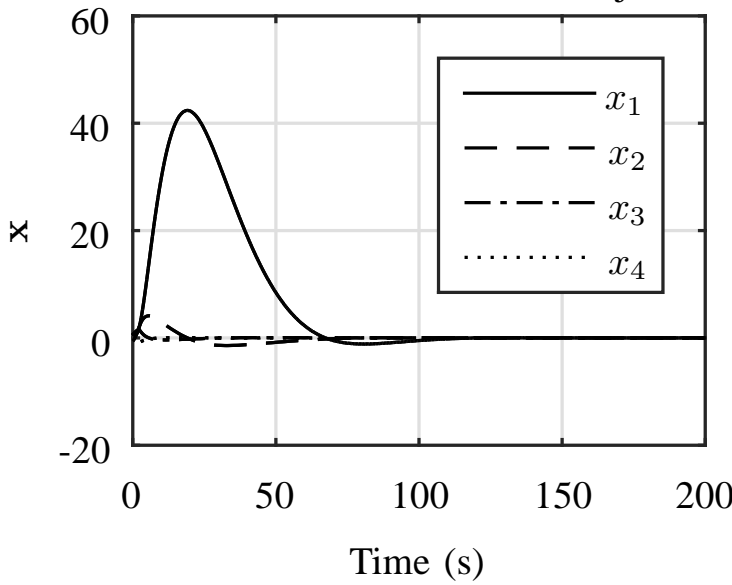

Control law without disturbance rejection

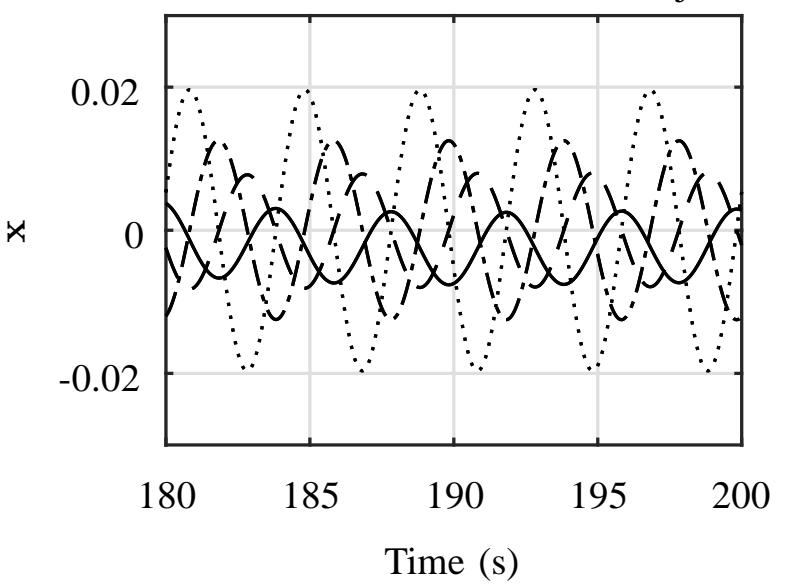

Control law with disturbance rejection

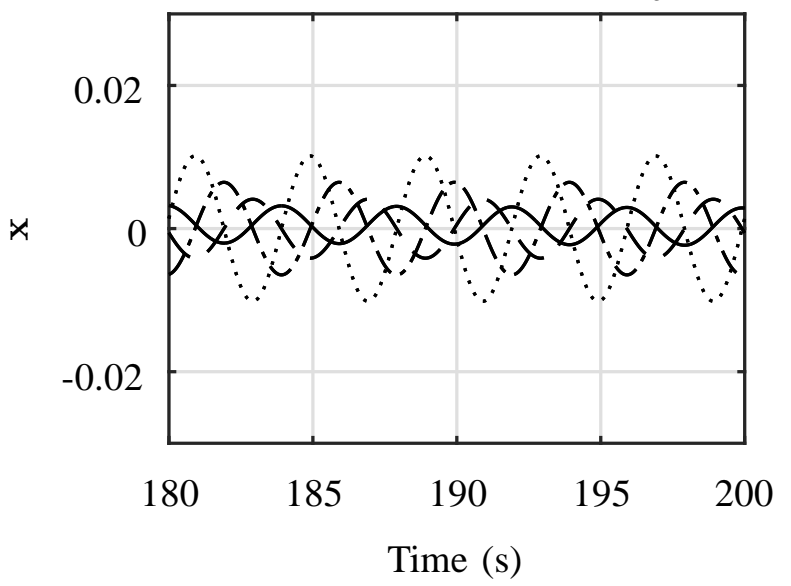

Figure 3. Controller with and without high-gain disturbance rejection.

By using exponential activation functions, it allows for quick convergence of the states in a successive manner performing better than the nested saturation method (at least for this set of gains). Additionally, the method can be extended to facilitate disturbance rejection through high gain feedback that can be activated once the states have converged to a bounded set close to the origin, thereby improving the performance of the proposed approach.

The objective of this paper has been to share the main idea of how to control a chain of integrators with limited actuation using exponential activation functions, and it can be considered future work to show the stability of the approach. Another item that can be considered future work, is to test the performance of the approach for controlling quadrotors (or another plant that can be described as a fourth order system) where the control law in [8] can be replaced with a new control law based on the results from this paper.

\section{REFERENCES}

[1] A. R. Teel, "Global stabilization and restricted tracking for multiple integrators with bounded controls," Systems \& Control Letters, vol. 18, pp. 165-171, 1992.
[2] J. Farrell, M. Sharma, and M. Polycarpou, "Backstepping-based flight control with adaptive function approximation," Journal of Guidance, Control, and Dynamics, vol. 28, no. 6, pp. 1089-1101, 2005.

[3] T. Lauvdal, R. Murray, and T. I. Fossen, "Stabilization of integrator chains in the presence of magnitude and rate saturations: A gain scheduling approach," in Proceedings of the IEEE Conference on Decision and Control, San Diego, USA, 1997.

[4] H. J. Sussmann, E. D. Sontag, and Y. Yang, "A general result on the stabilization of linear systems using bounded controls," IEEE Transactions on Automatic Control, vol. 39, no.12, pp. 2411-2425, 1994.

[5] L. Marconi and A. Isidori, "Robust global stabilization of a class of uncertain feedforward nonlinear systems," Systems \& Control Letters, vol. 41, pp. 281-290, 2000.

[6] S. Tarbouriech, G. Garcia, J. M. G. da Silva Jr., and I. Queinnec, Stability and stabilization of linear systems with saturating actuators. Springer, ISBN 978-0-85729-940-6, 2011.

[7] S. Gayaka and B. Yao, "Global stabilization of a chain of integrators with input saturation and disturbances," in Proceedings of the American Control Conference, San Francisco, CA, USA, 2011.

[8] E. Oland, "A command-filtered backstepping approach to autonomous inspections using a quadrotor," in Proceedings of the 24th Mediterranean Conference on Control and Automation, (Athens, Greece), 2016.

[9] R. Schlanbusch and R. Kristiansen, "Control of spacecraft formation with disturbance rejection and exponential gains," Modeling, Identification and Control, vol. 34, no. 1, pp. 11-18, 2013.

[10] E. N. Johnson and S. K. Kannan, "Nested saturation with guaranteed real poles," in Proceedings of the American Control Conference, Denver, Colorado, USA, 2003. 